\title{
Self-mixing Sensor for Real-time Measurement of Harmonic and Arbitrary Displacements
}

\author{
U. Zabit ${ }^{1-2}$, O.D. Bernal ${ }^{1-2}$, and T. Bosch ${ }^{1-2}$ \\ ${ }^{1}$ CNRS, LAAS, 7 avenue du colonel Roche, F-31400 Toulouse, France \\ ${ }^{2}$ Univ de Toulouse, INP, LAAS, F-31400 Toulouse, France
}

\begin{abstract}
A real-time self-mixing laser displacement sensor is presented that uses a fast consecutive-samples based unwrapping algorithm. The processing is achieved at a rate of $125 \mathrm{KHz}$ by an integrated micro-converter (ADuC7020) embedded on the SM sensor. Arbitrary and harmonic displacements have been measured in real-time with $\lambda / 10$ precision as compared with the reference built-in capacitive feedback sensor of the target, where $\lambda$ is the laser wavelength. The algorithm does not require any parameter estimation or huge memory and corrects possible false fringe detections. This results in a compact, integrated, precise and self-aligned laser sensor that costs less than 40 \$.
\end{abstract}

Keywords: real-time, displacement sensor, self-mixing interferometry, optical sensor.

\section{SELF-MIXING INTERFEROMETRY}

Self-mixing (SM) or optical feedback interferometry technique has been regularly employed during the last two decades for displacement, velocity, distance, flow and angle measurements as the resulting sensor is of a simple, compact, self-aligned, and low-cost type [1-2].

SM effect occurs in a laser when a part of the beam backscattered by a target is coupled back into the laser cavity and causes interference with the emitted beam, thus modifying the spectral properties of the laser. The variations in the optical output power of the laser diode $P(t)$ caused by this optical feedback can be written as [3]:

$$
P(t)=P_{0}\left[1+m \cdot \cos \left(x_{F}(t)\right)\right]
$$

where $P_{0}$ is the emitted optical power under free-running conditions, $m$ is the modulation index and $x_{F}(t)$ is the laser output phase in the presence of feedback, given by:

$$
x_{F}(t)=2 \pi \frac{D(t)}{\lambda_{F}(t) / 2}
$$

where $D(t)$ is the target displacement.

The emission wavelength subject to feedback $\lambda_{F}(t)$ is given by the phase equation:

$$
x_{0}(t)-x_{F}(t)-C \sin \left[x_{F}(t)+\arctan (\alpha)\right]=0
$$

where $\alpha$ is the linewidth enhancement factor, $C$ is the feedback coupling factor that determines the SM operating regime and $x_{0}(t)$ is the laser output phase in the absence of feedback, obtained by replacing $\lambda_{F}(t)$ with $\lambda$ in (2), where $\lambda$ is the laser diode emission wavelength under free running conditions.

A typical SM signal obtained for an arbitrary target movement is presented in Fig. 1. The built-in photodiode available in the laser diode package can be used to retrieve the SM signal. Each fringe in the SM signal represents a target displacement of $\lambda / 2$. So, by using simple fringe counting, a sensor would ensure $\lambda / 2$ precision by detecting all fringes. However, various algorithms exist that allow even better measurement precision [3-5].

Before elaborating the operating principle of the present sensor, let us first look at some of the published real-time SM sensors.

\section{REAL-TIME SM DiSPLACEMENT SENSORS}

As stated above, a basic SM sensor with a $\lambda / 2$ precision can be developed by using real-time simple fringe counting. Such is the case for the real-time three degrees-of-freedom motion sensor described in [6].

However, better precision real-time SM sensors have been recently published. For example, the fully analog SM vibration sensor [5] indicates a measurement precision of $35 \mathrm{~nm}$. This innovative analog design is based on the unwrapping of the SM signal, a technique which is also used in our present sensor. This sensor, however, relies on an analog integrator stage whose drift can disturb the displacement retrieval as seen in Fig. 4 of [5]. Similar issues may have inhibited the use of this sensor for arbitrarily varying target motion.

Likewise, another novel reconstruction method for vibration measurements has been proposed in [7] that can exploit SM signal even in weak-feedback regime. The method is, however, designed for a harmonic motion of the target and no arbitrary target displacements results were presented. 


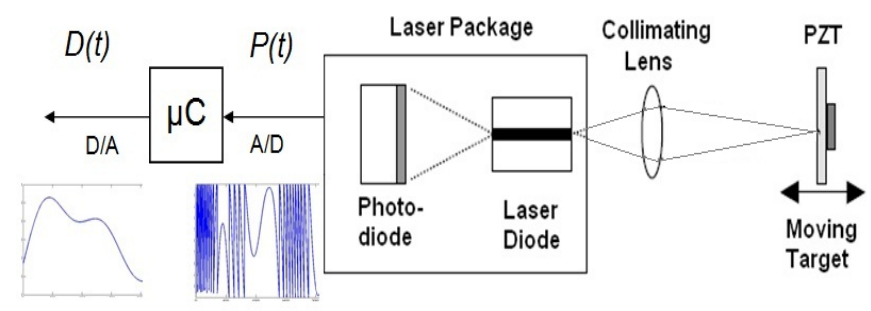

Figure 1. Schematic diagram of the embedded real-time self-mixing displacement sensor with a micro-converter $(\mu \mathrm{C})$.

So, in comparison with these real-time SM displacement sensors, our present sensor provides precise measurements for both arbitrary and harmonic target movements. The digital processing also ensures that the sensor is not affected by any drift issues. The operating principle of the sensor is now elaborated in the following section.

\section{UNWRAPPING OF SELF-MIXING SIGNAL}

In order to achieve a better than $\lambda / 2$ precision, further information can be extracted from a SM signal by following the shape of each SM fringe. It has been previously shown that the shape of the SM fringe is indicative of the actual target movement [8]. In fact, if it weren't for the phase jumps occurring for every $\lambda / 2$ target displacement, the SM signal would remain indicative of the target movement. This idea has thus been exploited to propose SM displacement measurement techniques with better precision, such as [3-5]. Note that these techniques thus follow the SM signal shape as opposed to the technique presented in [9] that uses local linearization of SM signal shape while compensating possible fringe-loss of the SM signal.

Let us now look into one of these unwrapping techniques called the phase unwrapping method having a precision of $\lambda / 16$. It is summarized below and would be subsequently used for comparing the results of our proposed method that is detailed in the following sub-section.

\section{A. $\quad$ Phase Unwrapping Method}

The Phase Unwrapping Method (PUM) has two principal steps [3]. The first one leads to the rough estimation of the phase $x_{F}(t)$. The output of this first step, guaranteeing at least a $\lambda / 2$ precision, is similar to the final output of the sensor presented in [6] or the first stage output of the sensor presented in [5]. The second signal processing step deals with the joint estimation of parameters, $C$ and $\alpha$, by running an optimisation routine. After the parameter estimation, the use of phase equation (3) allows to retrieval of the resultant phase $x_{0}(t)$, which is then used to finally recover the displacement $D(t)$.

Let us now look at the SM signal processing approach used in our present set-up.

\section{B. Consecutive-Samples based Unwrapping}

The present algorithm, called the Consecutive-Samples based Unwrapping (CSU) produces an output similar to the output of the first stage of the PUM. As already detailed, the shape of the SM fringe represents the target movement except for the instants where we observe sharp discontinuities indicative of a $\lambda / 2$ target displacement. Thus, a signal representative of the target movement can be obtained by unwrapping these discontinuities while adding the instantaneous SM signal value to the output.

The processing steps can be seen in Fig. 2. For a given SM signal (Fig. 2 a), we start by detecting the positive or negative fringes contained in the SM signal (Fig. 2 b). A staircase signal (Fig. 2 c) is then obtained by a continuous addition/subtraction of a step by using the SM fringe detection information. The amplitude of the step of the staircase signal is thus equivalent to a $\lambda / 2$ target displacement. Finally, just as in [5], we only need to add the scaled SM signal to the staircase signal to obtain the retrieved signal proportional to target movement (Fig. 2 d). The scaling/normalization of the SM signal (Fig. 2 a) is necessary so that the peak-to-peak amplitude of the scaled SM signal matches the amplitude of the step chosen for the staircase signal [5]. A lack of matching between the scaled SM signal and the step would result in error in displacement measurement.

What is interesting in this approach is the fact that we need only process two consecutive SM signal samples at a time in order to continuously reconstruct the target displacement. Thus, with a delay of only one sample, the output can be updated. A running derivative based on $P(t)-P(t-1)$ is compared with a threshold to detect positive or negative fringes. This value updates an accumulator (resulting in the staircase signal) to which the present value $P(t)$ is added resulting in the output displacement $D(t)$. Thus, each incoming SM sample results in a corresponding output displacement sample.
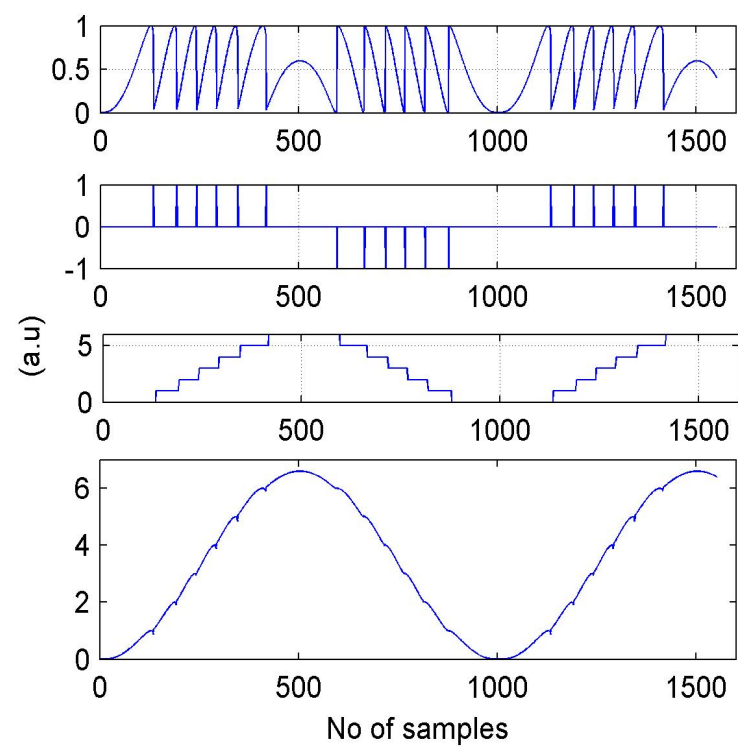

Figure 2. Displacement retrieval through Consecutive-Samples based Unwrapping. (a) normalized SM signal corresponding to $C=1.5$, (b) fringe discontinuity detection, (c) staircase signal representing the fringe count and (d) the retrieved signal proportional to target excitation signal obtained by the addition of signals (a) and (c). 

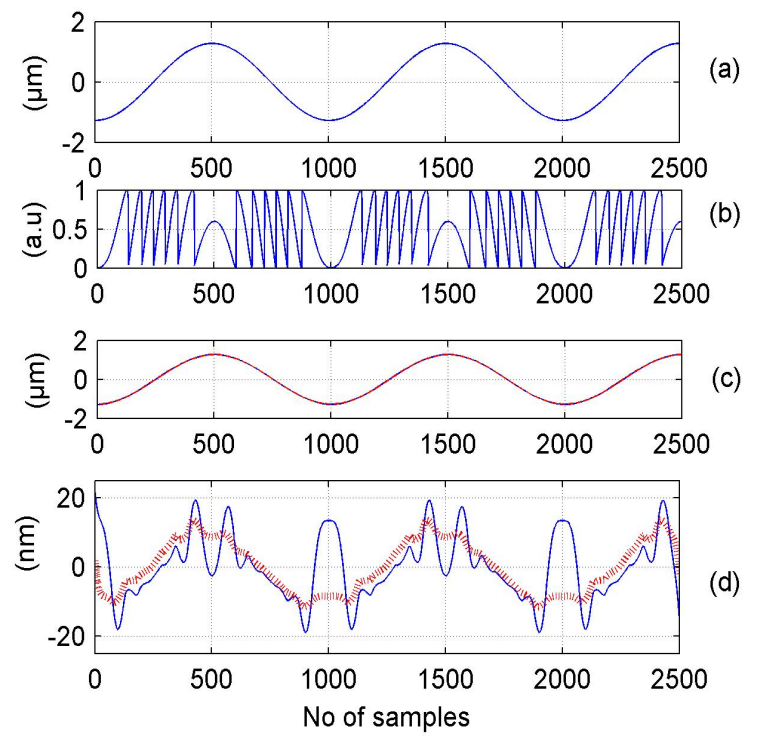

Figure 3. Simulation of sinusoidal displacement measurement. (a) target displacement, (b) corresponding SM signal, (c) displacement retrieval using CSU (blue) and PUM (dotted red) and (d) error with respect to simulated target displacement for CSU (blue) and PUM (dotted red).

As compared to a complex approach such as the PUM that is based on joint-estimation of SM parameters requiring iterative optimization routines, the consecutive-sampled unwrapping has the great benefit of requiring only two SM samples to update the corresponding displacement output. This lack of parameter estimation allows a fast real-time displacement measurement. It will be seen below that the ensuing results, though not as precise as the final output of PUM, still compare favorably with the PUM displacement.

\section{Comparison between CSU and PUM}

Assuming a laser with $\lambda=785 \mathrm{~nm}$, the displacements obtained by using these two methods have been compared with the simulated target displacement for arbitrary and harmonic target movements.

\section{1) Simulated Harmonic Displacementt}

Fig. 3 presents the results where it can be seen that the error for CSU is slightly higher as compared with that for PUM. However, the real-time nature of CSU is a huge advantage as compared to the computationally heavy PUM. Max CSU error is less than $20 \mathrm{~nm}$ for a micrometric target movement.

\section{2) Simulated Arbitrary Displacement}

Fig. 4 presents the results for an arbitrary simulated target displacement. The errors of both methods are again comparable and that of CSU remains less than $50 \mathrm{~nm}$.

\section{EXPERIMENTAL SET-UP}

The experimental SM displacement sensor set-up used to validate the CSU method has been presented in Fig. 1. Here, a commercial PZT (Piezo-electric Transducer) from Physik Instrumente (P753.2CD) is used as the target. The PZT is equipped with a built-in capacitive feedback sensor (CFS) for direct-motion metrology with a precision of $2 \mathrm{~nm}$. The CFS is used as a reference sensor in order to compare the output of real-time CSU based displacement measurement.

The SM sensor is based on a laser diode package from Sanyo (DL7140) emitting at $785 \mathrm{~nm}$. The integrated microconverter $(\mu \mathrm{C})$ is the AduC7020 from Analog Devices ${ }^{\circledR}$ working at $40 \mathrm{MIPS}$. The $\mu \mathrm{C}$ has integrated Analog-to-Digital (A/D) and Digital-to-Analog (D/A) converters. The incoming signal $P(t)$ is processed into the output displacement signal $D(t)$ at $125 \mathrm{KHz}$. So, the output displacement is updated every $8 \mu \mathrm{s}$.

The analog output of the DAC has a linear range of $2.8 \mathrm{~V}$. So, in order to guarantee a $\lambda / 10$ precision for a maximum target displacement of $30 \mu \mathrm{m}$, we set the step amplitude to $\pm 36 \mathrm{mV}$ (corresponding to a $\pm \lambda / 2$ target displacement). This then necessitates that the SM signal at the input of the ADC needs to be digitally scaled to [0 $36 \mathrm{mV}]$ range by the micro-converter in order to match the scaled/normalized SM signal to the chosen step amplitude. The real-time SM displacement sensor sensitivity at the output of the micro-converter DAC is thus of $91.72 \mathrm{mV} / \mu \mathrm{m}$.

As the photodiode output providing the SM signal can be finally scaled to $[036 \mathrm{mV}$ ] range so no other calibration of the system is needed and it is able to update the target displacement measurement based on two consecutive SM samples (needed for derivation leading to SM fringe detection).

Experimental data has showed that 20 samples per SM fringe are normally needed in order to correctly detect the fringe-discontinuity of each SM fringe having its associated noise. This then provides the maximum measurable target speed of $2.45 \mu \mathrm{m} / \mathrm{ms}$ for the real-time displacement sensor.

Let us now look into the working of the micro-converter based sensor for experimental real-time SM signal processing.
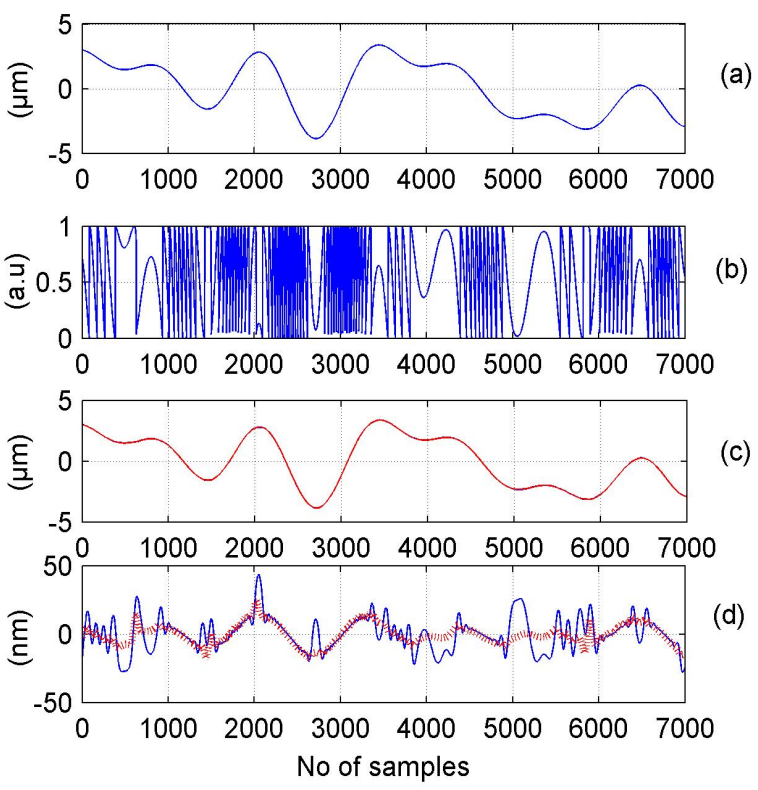

Figure 4. Simulation of arbitrary displacement measurement. (a) target displacement, (b) corresponding SM signal, (c) displacement retrieval using CSU (blue) and PUM (dotted red) and (d) error with respect to simulated target displacement for CSU (blue) and PUM (dotted red). 


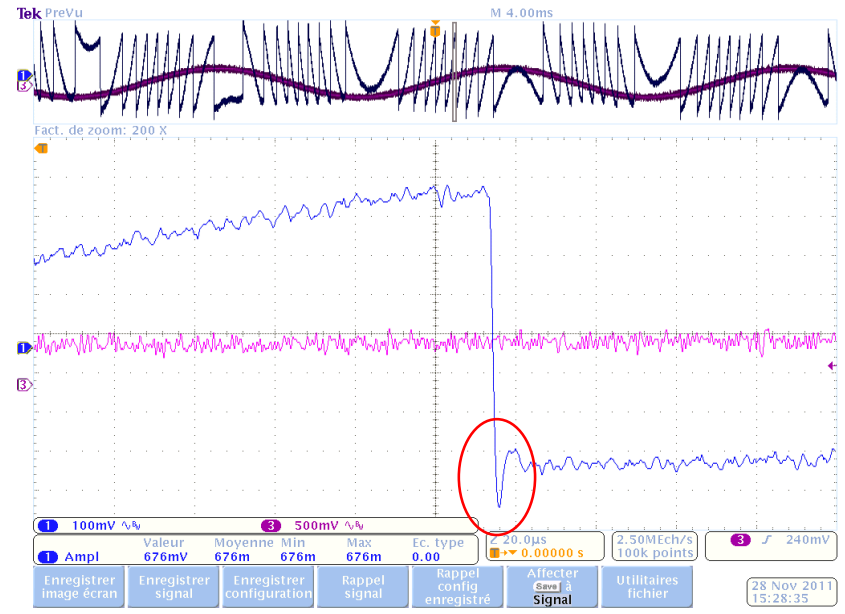

Figure 5. The ringing or "sparkle" at the tip of the SM fringe. It may lead to a false fringe detection that needs to be corrected. PZT mouvement (red) and corresponding SM signal (blue).

\section{A. Correction of False Fringe Detection}

The CSU method incorporates correction of possible false fringe detection. As each false fringe detection would introduce an error of $\lambda / 2$ so such a correction is of utmost importance in order to guarantee a precise measurement. Two cases that may lead to false fringe detection are presented below.

\section{1) Ringing of the SM fringe}

For an experimental SM signal, it may happen that the SM fringe exhibits a ringing or "sparkle" [10] at the end of the discontinuity (as seen in Fig. 5). Such a sharp reversal just after the discontinuity can result in a false detection (true +1 fringe detection followed just after by -1 detection or vice-versa).

The processing proposed in [10] aims to remove such a "sparkle" from the SM signal by using a combination of Kaiser and Median filters. In our case, the CSU method guarantees against such a false detection by not considering the subsequent sample that arrives just after a sample that has lead to a positive or a negative fringe detection. Thus, instead of filtering (at a cost of increased computations) the SM signal, it is preferred to block the detection for possibly suspect samples.

\section{2) Multiple Sampling of Fringe Discontinuity}

It may happen that the SM fringe discontinuity is sampled more than once (Fig. 6) by the A/D converter. For the case shown in Fig. 7, the amplitude of the derivative signal gets distributed over two samples. This in turn necessitates a lowering of the threshold value used to distinguish fringe discontinuity. However, now, two fringes may get detected where only one exists in the experimental signal (indicated by a sequence of $+1+1$ or $-1-1$ over two consecutive samples). Such a false detection is again avoided by the CSU method by discarding the second detection.

\section{B. Experimental Real-time Results}

The real-time SM displacement sensor has been used to measure arbitrary and harmonic displacements. The CFS was used to compare the real-time sensor results. The screenshots of acquisitions and error results are presented below.

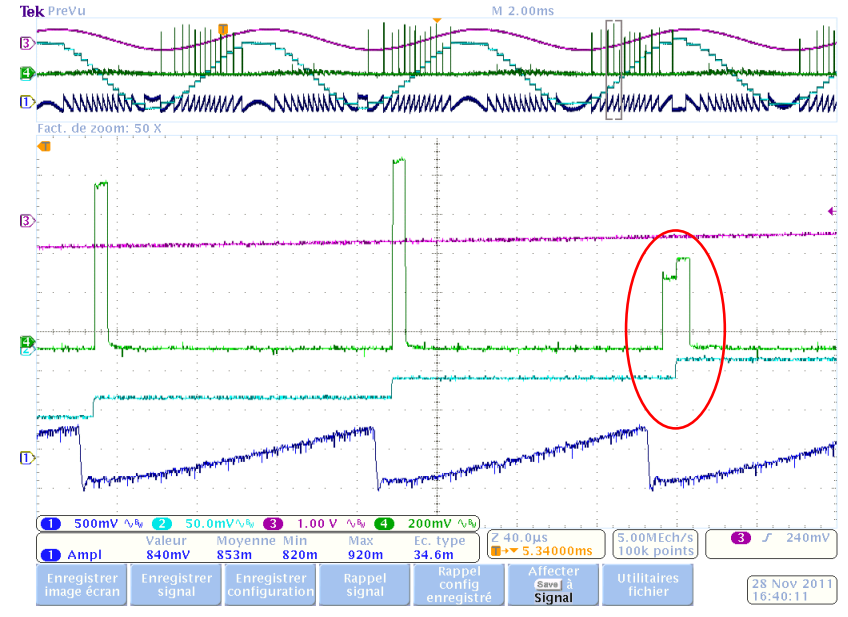

Figure 6. Influence of sampling on the derivative signal used for fringe detection. Here the right-most fringe discontinuity has been sampled twice. It thus necessitates lowering of threshold as well as removal of false detection. SM signal (violet), derivative signal (green) and staircase signal (light blue).

\section{1) Harmonic Real-time Displacement Measurement}

The PZT was excited with a $70 \mathrm{~Hz}$ sinusoidal signal of 5 $\mu \mathrm{m}$ amplitude. The corresponding real-time displacement retrieval is presented in Fig. 7. It is seen that the real-time CSU based displacement signal (green curve) resembles closely the PZT movement indicated by the CFS (purple curve) as compared with the staircase signal (light blue curve).

The difference between the CSU based signal and the CFS signal has been plotted in Fig. 8 (blue curve). The off-line displacement measurement based on PUM is also plotted (dotted red curve) for comparison's sake. It can be verified that the real-time CSU result is quite close to PUM based result obtained by processing the corresponding SM signal (Fig. 8 violet curve) on a computer. The relatively higher error for experimental signals as compared with the error for simulated signals (Fig. 3) may be explained by possible misalignment between the laser sensor and PZT and the presence of noise.

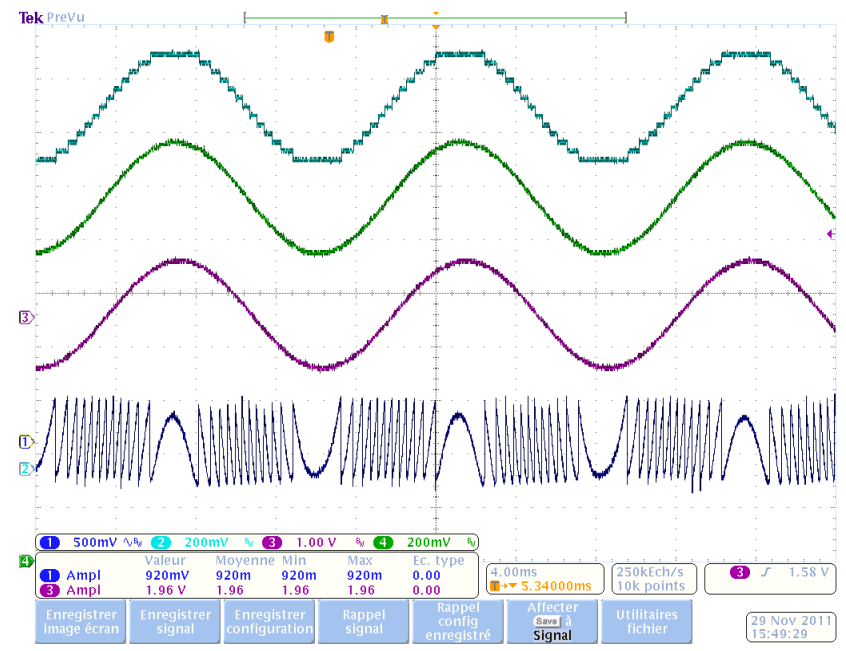

Figure 7. Real-time sinusoidal displacement measurement of PZT vibrating at $70 \mathrm{~Hz}$ with a $5 \mu \mathrm{m}$ p-p amplitude. CFS reference displacement (purple), corresponding SM signal (violet), real-time staircase signal (light blue) and real-time CSU displacement signal (green). 


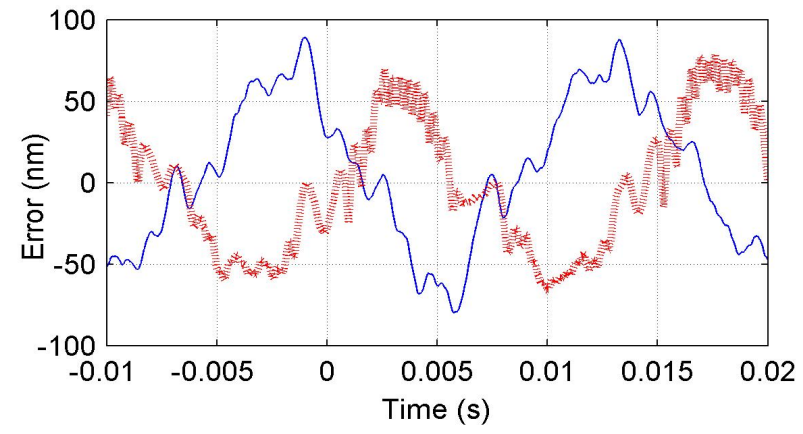

Figure 8. Error results of retrieved displacements with respect to CFS for the signals corresponding to the $70 \mathrm{~Hz}$ PZT excitation. Error for the real-time CSU based signal (blue). Error for the off-line PUM based signal (dotted red).

\section{2) Arbitrary Real-time Displacement Measurement}

The experimental real-time displacement measurement for an arbitrary target movement is shown in Fig. 9. Once again, the CSU based displacement (light blue curve) is quite close to CFS displacement (purple curve).

\section{ACKNOWLEDGMENT}

The authors acknowledge the technical support provided by Clément Tronche and Francis Jayat for electronic circuit boards and experimental set-up.

\section{CONCLUSION}

In this paper, a fast real-time self-mixing embedded displacement sensor with $\lambda / 10$ precision is presented. The sensor is based on Consecutive-Samples based Unwrapping method that allows precise results while processing only two incoming samples at a time. This approach has been simulated and compared with a previously published Phase Unwrapping Method and provides comparable results. The algorithm has been deployed on an integrated micro-converter (ADuC7020) coupled with the SM sensor that updates the output displacement within $8 \mu \mathrm{s}$. The sensor has been experimentally validated for arbitrary and harmonic displacements of a target with a built-in reference sensor of $2 \mathrm{~nm}$ precision. This results in a compact and self-aligned laser displacement sensor guaranteeing $\lambda / 10$ precision that costs less than $40 \$$.

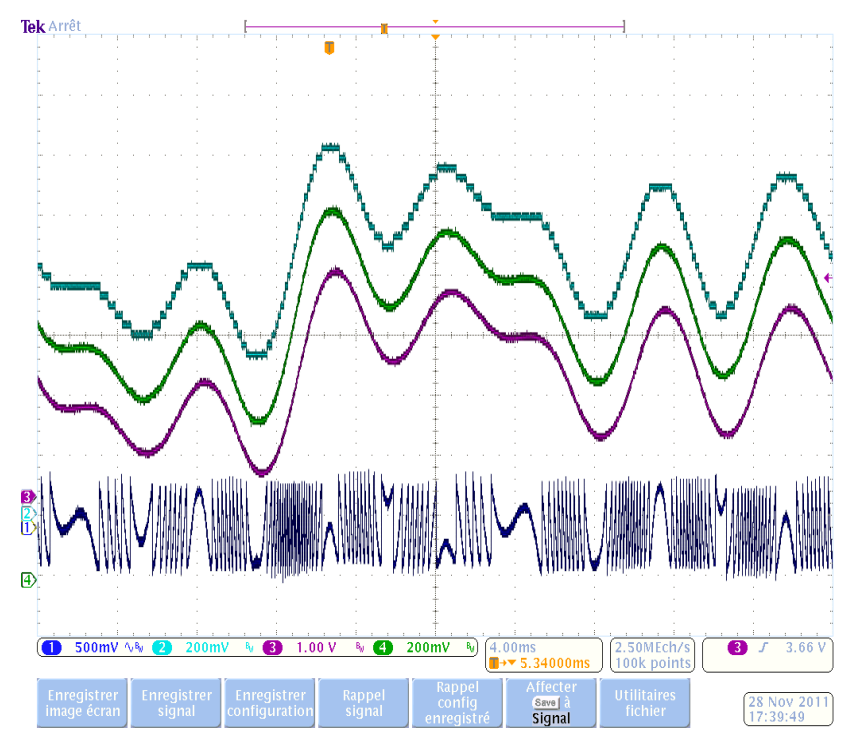

Figure 9. Real-time arbitrary displacement measurement of PZT. CFS reference displacement (purple), corresponding SM signal (violet), real-time CSU based signal (light blue) and real-time CSU displacement signal (green).

\section{REFERENCES}

[1] S. Donati, "Electro-Optical Instrumentation - Sensing and Measuring with Lasers", Prentice Hall, 2004.

[2] T. Bosch, C. Bes, L. Scalise, and G. Plantier, "Optical feedback interferometry," Encyclopedia of Sensors, American Scientific Publishers, vol. 7, pp. 107-127, 2006.

[3] C. Bes, G. Plantier and T. Bosch, " Displacement measurements using a self-mixing laser diode under moderate feedback", IEEE Trans. Instrum. \& Meas. Vol. 55, pp.1101, 2006.

[4] Yuanlong Fan, Yanguang Yu, Jiangtao $\mathrm{Xi}$, and Joe F.Chicharo, "Improving the measurement performance for a self-mixing interferometry-based displacement sensing system", Applied Optics, Vol. 50, no 26,pp 5064-5072, Sept. 2011.

[5] Norgia, M.; Pesatori, A.;, "Fully analog self-mixing laser vibrometer," Inst. and Meas. Techn. Conf. (I2MTC), 2011 IEEE, pp.1-4, May 2011.

[6] Ottonelli, S.; De Lucia, F.; di Vietro, M.; Dabbicco, M.; Scamarcio, G.; Mezzapesa, F.P.; , "A Compact Three Degrees-of-Freedom Motion Sensor Based on the Laser-Self-Mixing Effect," Photonics Technology Letters, IEEE, vol.20, no.16, pp.1360-1362, Aug.15, 2008.

[7] Magnani, A.; Pesatori, A.; Norgia, M.; , "Novel displacement reconstruction method for vibration measurements," Inst. and Meas. Techn. Conf. (I2MTC), 2011 IEEE, vol., no., pp.1-4, 10-12 May 2011.

[8] Noël Servagent, Flore Gouaux and Thierry Bosch, "Measurements of displacement using the self-mixing interference in a laser diode", J. Opt. 29, pp. 168-173, 1998.

[9] Zabit, U.; Bony, F.; Bosch, T.; Rakic, A.D.; , "A Self-Mixing Displacement Sensor With Fringe-Loss Compensation for Harmonic Vibrations," Photonics Technology Letters, IEEE, vol.22, no.6, pp.410412, March15, 2010.

[10] Yanguang Yu; Jiangtao Xi; Chicharo, J.F.; "Improving the Performance in an Optical feedback Self-mixing Interferometry System using Digital Signal Pre-processing," Intelligent Signal Processing, 2007. WISP 2007. IEEE International Symposium on, vol., no., pp.1-6, 3-5 Oct. 2007. 